\title{
Los estudios del paisaje sonoro y la geografía cultural (1971-2020)
}

Recepción: 1 de mayo de 2021 - Evaluación: 20 de octubre de 2021 • Aprobación: 8 de noviembre de 2021

Resumen: En este trabajo realizamos un análisis descriptivo del desarrollo histórico conceptual del paisaje sonoro. El objetivo es identificar la manera en que se ha construido un campo de estudio alrededor de dicha propuesta. Se desarrolló una búsqueda selectiva de literatura que tuvo como punto de partida textos clave de Murray Schafer y del World Soundscape Project, a partir de los cuales se rastreó su influencia en distintas partes del mundo. Se identificó un periodo de expansión durante la década de 1980, en el cual se conformaron sus dos enfoques principales: el ecológico y el perceptual-cultural. Así mismo, se hizo énfasis en la manera en que fue abordado dicho término desde enfoques culturalistas en geografía y se identificó que a partir de las discusiones hechas surgieron como propuesta las geografías auditivas y del sonido. Finalmente, se realizó una revisión sistemática en índices y buscadores de literatura académica y se encontró un interés latente, durante los últimos tres lustros, por incorporar el sonido como objetivo de estudio a partir de alguna de las posturas mencionadas (paisaje sonoro, geografias auditivas y geografías del sonido), sin embargo, es un tema que continúa en proceso de consolidación.

Palabras clave: geografia humana, paisaje, percepción, paisaje sonoro.

Para citar: Garrido Rojas, D., \& Urquijo Torres, P. S. (2022). Los estudios del paisaje sonoro y la geografía cultural (1971-2020). Perspectiva Geográfica, 27(1), 51-68. https://doi. org/10.19053/01233769.12797

1 Maestro en Geografia, Universidad Nacional Autónoma de México. Estudiante de doctorado en el Posgrado en Geografia Centro de Investigaciones en Geografía Ambiental, Universidad Nacional Autónoma de México. Artículo realizado con la beca de estudios de doctorado del Consejo Nacional de Ciencia y Tecnología, México. Correo: dgarrido@pmip.unam.mx. Orcid: https://orcid.org/0000-0002-0669-5013

Doctor en Geografía, Universidad Nacional Autónoma de México. Investigador titular en el Centro de Investigaciones en Geografĩa Ambiental, Universidad Nacional Autónoma de México. Correo: psurquijo@ciga.unam.mx. Orcid: https://orcid. org/0000-0001-9626-0322 


\title{
Soundscape studies and cultural geography (1971-2020)
}

\begin{abstract}
In this paper, we carry out a descriptive analysis of the historical conceptual development of soundscape. The aim is to identify how a study environment has been built around this proposal. We developed a selective search of the literature, starting with key texts by Murray Schafer and the World Soundscape Project, from which we traced its influence in different parts of the world. A period of expansion was identified during the 1980 s, in which its two main approaches were shaped: the ecological and the perceptional-cultural. Likewise, special emphasis was placed on how the term was approached from the culturalist perspective in geography, and it was identified that from these discussions arose the proposal of auditive geographies and sound geographies. Finally, a systematic review was carried out in indexes and search engines of academic literature and we found a latent interest, during the last fifteen years, in incorporating sound as an objective of study from some of the positions mentioned (soundscape, auditive geographies, and sound geographies), however, it is a topic that is still in the process of consolidation.
\end{abstract}

Keywords: human geography, landscape, perception, soundscape.

\section{Os estudos da paisagem sonora e a geografia cultural (1971-2020)}

\begin{abstract}
Resumo: Este trabalho realizamos uma análise descritiva do desenvolvimento histórico conceitual da paisagem sonora. O objetivo é identificar a forma como foi-se construído um campo de estudo em torno desta proposta. Desenvolveu-se uma procura seletiva de literatura que teve como ponto de partida textos chave de Murray Schafer e do World Soundscape Project, a partir dos quais se localizou sua influência em diferentes partes do mundo. Identificouse um período de expansão durante a década de 1980, no qual conformaram-se suas duas abordagens principais: o ecológico e o perceptual-cultural. Além disso, se fez uma ênfase no modo como este termo foi abordado a partir de abordagens culturalistas em geografia e foi identificado que a partir das discussões feitas surgiram como proposta as geografias auditivas e do som. Finalmente, realizou-se uma revisão sistemática em índices e buscadores de literatura acadêmica e achou-se um interesse latente, durante os últimos três lustros, por incorporar o som como objetivo de estudo a partir de alguma das posturas mencionadas (paisagem sonora, geografias auditivas e geografias do som), no entanto, é um tema que continua em processo de consolidação.
\end{abstract}

Palavras-chave: geografia humana, paisagem, percepção, paisagem sonora. 


\section{Introducción}

En las últimas décadas del siglo xx, con la difusión de los giros culturales en geografia (Barnett, 1998; Hiernaux, 2010; Ferreti, 2019), se presentó un mayor interés por el análisis de otras formas de aproximación al paisaje, alternativas al predominio de lo visual. Si bien es cierto que la observación del paisaje ha estado en la geografía desde sus inicios, fundamentada en el análisis de la fisonomía visible de la Tierra y sus organizaciones subyacentes, los enfoques recientes reconocen otros canales sensoriales que complementan, o compensan, la importancia de lo que se mira (Rose, 2003). Particularmente, el paisaje como sonido ha sido objeto de estudio a través de lo que se ha denominado paisaje sonoro. No obstante que las investigaciones pioneras sobre el tema aluden a áreas espaciales que remiten a la potencial distribución y conectividades del sonido (Schafer, 1977; Truax, 1978), no existió una postura fundamentalmente geográfica para su análisis. Pese a lo anterior, el concepto fue retomado desde distintos enfoques geográficos, entre ellos, el cultural. En este texto pretendemos plantear las tendencias actuales de investigación respecto al paisaje sonoro desde ese enfoque en particular.

Raymond Murray Schafer (1977; 2006) fue pionero en el estudio del paisaje sonoro, quien desde la década de los setenta estableció su carácter interdisciplinario y las posibilidades de abordaje desde muy distintos ámbitos, tanto sociales y ecológicos como artísticos y humanísticos. Schafer postuló que el paisaje sonoro (soundscape) era el campo resonante total de un lugar, caracterizado por un dinamismo constante, pues cambiaba incluso con las horas del día. Además, involucraba necesariamente aquello que un individuo o un grupo de personas percibían e interpretaban, por lo que el análisis del paisaje sonoro incluyó las abstracciones que los sujetos realizaban respecto a los sonidos de los lugares (Truax, 1978).

El tema central del paisaje sonoro, entonces, no es únicamente el sonido como elemento físico - como lo es, por ejemplo, para la acústica-, sino la manera en que culturalmente es escuchado e interpretado en contextos geográficos específicos. El término también hace alusión a una comprensión histórica, a través del registro que lo pudiera preservar; por ejemplo, composiciones musicales, sus descripciones literarias, las grabaciones de audio o el acomodo de estas, a manera de montaje, con el fin de mostrar las características audibles representativas de un sitio, entre otras (Truax, 1978). En este sentido, Emily A. Thompson (2002) hace hincapié en el papel de la cultura para comprender el paisaje sonoro, poniendo el énfasis en los seres humanos en la conformación de este y las distintas maneras en que perciben el sonido a partir de distintos lugares. En síntesis, el paisaje sonoro no se expresa únicamente a partir de su componente físico - los sonidos-, sino que, como cualquier paisaje, es resultado de un constructo cultural y se encuentra interrelacionado por todo aquello que una sociedad define.

El concepto paisaje alude a una unidad de terreno dinámicamente modelada por factores tanto socioculturales como biofísicos, el cual es abstraído a través de los distintos canales de percepción sensorial de quienes lo habitan o lo interpretan (Urquijo \& Barrera, 2009; Antrop \& Eetvelde, 2019; Olwig, 2019). El paisaje es una expresión física y representacional de la relación sociedad-naturaleza, guiada por procesos imaginativos, creativos u emocionales (Cosgrove, 2002; Taylor, 2008). El paisaje se expresa así a través de valoraciones estéticas y posicionamientos éticos compartidos en sociedad, que lo definen y transforman históricamente. Cuando en el estudio del paisaje se enfatizan estas condicionantes culturales, se establece un posicionamiento reflexivo respecto al reconocimiento de los valores, conocimientos o sentimientos que las diferentes sociedades poseen sobre sus lugares y las formas de transformarlos y vivirlos (Urquijo, 2021). En este sentido, la sonoridad como una manifestación de la experiencia sensorial que guía el modelado o representación de paisajes es un tema más que pertinente desde la ciencia geográfica. 
Es importante señalar que el paisaje sonoro se interpreta en dos sentidos. Por una parte, como una fracción de espacio cuya sonoridad es resultado de los procesos biofísicos y socioculturales que allí ocurren. El carácter o personalidad del paisaje sonoro se define por las particularidades históricas y culturales de quien o quienes escuchan. Por otra parte, paisaje sonoro también es una manera de referirse al campo de estudio, articulando investigaciones de diversas disciplinas, con sus propias construcciones teóricas y metodológicas, aunque no necesariamente establezcan consideraciones espaciales o territoriales.

Con fines de concreción, en este artículo nos centramos en los trabajos que se elaboran desde el enfoque cultural en geografía, reconociendo en ellos la importancia que se otorga a los aspectos sensoriales y vivenciales en el estudio del paisaje (Mitchell, 1995; Claval, 1999; Kramsch, 1999). Asimismo, establecemos como punto de partida los trabajos que se empezaron a desarrollar en 1980, tras la fundación del World Soundscape Project. Ello establece una contextualización panorámica basada, sobre todo, en las corrientes anglosajona y francófona. Sin embargo, reconocemos que esto es solo el principio de una historia que, en tiempos más recientes, muestra múltiples caminos teóricos y operacionales en el estudio del paisaje sonoro y desde distintas regiones del mundo.

\section{Procedimiento metodológico}

En un primer momento, se realizó la búsqueda y revisión de bibliografía, considerando como punto de partida contextual la creación del World Soundscape Project en 1971 y su innovadora propuesta de estudios de entornos acústicos. Se revisaron los trabajos de $\mathrm{Mu}$ rray Schafer y Barry Truax y se rastrearon los autores y grupos de trabajo que se vieron influenciados por sus propuestas fundacionales. Los textos se ordenaron de manera cronológica. En una segunda etapa, se analizaron los principales trabajos que, desde la geografía cultural, fueron pioneros en la discusión del término desde sus propios referentes, fundamentalmente espaciales o territoriales. En este caso, el análisis consistió en identificar argumentos sobre la pertinencia de estudiar el paisaje sonoro y de qué manera aportaban al enfoque, así como las formas de emplearlo y las alternativas y soluciones que propusieron en sus críticas.

La tercera etapa tuvo como finalidad esbozar un panorama actualizado de trabajos respecto al paisaje sonoro. Para ello se realizó una búsqueda sistemática de artículos científicos desde el año 1995 al 2020. Como herramientas de búsqueda se emplearon los índices Scopus, Web of Science y los buscadores de literatura académica JSTOR y Redalyc. Se seleccionaron estos buscadores considerando que garantizaban una muestra más amplia de publicaciones a partir de los criterios seleccionados. En particular, Redalyc se eligió al concentrar publicaciones de la región latinoamericana. Con la finalidad de conocer las investigaciones publicadas en el mundo anglófono e hispanohablante se usaron palabras clave en español e inglés: paisajes sonoros, geografía cultural y geografía humana. Se decidió incluir esta última clave considerando que existe un espectro de trabajos dentro de la geografia humana que pudieron analizar el paisaje sonoro desde una postura diferente al enfoque culturalista. Las palabras clave se combinaron mediante operadores booleanos en los índices y buscadores salvo en Redalyc, cuyo motor de búsqueda no permitió dicha acción. Por lo tanto, en este último caso se usaron como palabras clave soundscape y paisaje sonoro. Se descartaron aquellos documentos que se enfocaron en aspectos que respondían a objetivos de otras disciplinas como acústica, musicología, literatura, comunicación, ciencias de la salud, legislación y los que no tuvieran una consideración propiamente geográfica, es decir, los que no analizaban la información a partir de posicionamientos espaciales o territoriales. En la Figura 1 se muestran los flujos de búsqueda, el número de artículos arrojados y los criterios específicos de selección. 
En total, 17 artículos fueron considerados para una revisión a profundidad debido a que contenían una amplia información tanto conceptual como explicativa del contexto de los paisajes sonoros. Se generó una base de datos donde se capturaron, además de los datos de referencia, área y periodo de estudio, los métodos empleados, el argumento central, la síntesis de los resultados y la síntesis de las conclusiones. Asimismo, se analizaron los referentes teóricos que sustentaron las investigaciones. Para ello se identificó la influencia de los trabajos pioneros en el tema, a qué corrientes dentro de la geografía cultural se hacía alusión y también se identificaron sus argumentaciones sobre la manera en que la geografía cultural se ve enriquecida con discusiones derivadas del análisis del paisaje sonoro.

\section{Contextos de surgimiento del campo de estudio: Canadá y Francia}

El punto de partida que consideramos pertinente para hablar del paisaje sonoro como campo de estudio es la formalización del World Soundscape Project en 1971 (Torigoe, 1982; Truax, 1978), iniciativa que estuvo integrada principalmente por compositores interesados en la grabación y composición musical a partir de sonidos ambientales, cuyos trabajos resultaron en aportes teóricos, metodológicos y de preservación y catalogación de sonidos. El surgimiento de dicha iniciativa debe entenderse vinculado al contexto social e intelectual que durante la década de los 1960 se suscitó en Occidente. Al respecto vale la pena rescatar un par de procesos ocurridos. Primero, en las ciudades existieron movimientos sociales y culturales conformados principalmente por jóvenes que cuestionaron los modos de vida derivados de las formas de producción industrial y que además reclamaban mayores derechos y libertades (Hobsbawn, 1995). El segundo fue un aumento en la conciencia y las acciones ante la inminente crisis ambiental, lo cual derivó en el surgimiento de organizaciones de alcance global como Greenpeace en Vancouver, Canadá, y cuyas primeras acciones fueron protestas en contra de las pruebas nucleares que el Gobierno estadounidense realizaba en Alaska (Eden, 2004).

En ese contexto, en 1965 se fundó en Vancouver la Simon Fraser University. Entre los objetivos de la institución estaba el establecer una distancia con los modelos educativos tradicionales; para ello implementó programas interdisciplinarios que respondieran a las circunstancias del momento histórico (Marontate et al., 2016). A Simon Fraser llegó Murray Schafer y ocupó el puesto de profesor residente en música, asociado a la Facultad de Educación. A través de la práctica docente, Schafer identificó el aumento de la contaminación acústica y la disminución en la conciencia auditiva de sus estudiantes, por lo que generó una serie de estrategias didácticas para entrenar la escucha (Schafer, 1967). En 1968 cambió su adscripción de la Facultad de Educación por el Departamento de Estudios de la Comunicación, en donde encontró la posibilidad de establecer el Sonic Research Studio, el cual se convirtió en la sede del posterior World Soundscape Project (Torigoe, 1982).

En el World Soundscape Project caracterizaron paisajes sonoros mediante la grabación de audio, el análisis de la intensidad de sonidos y su periodicidad de ocurrencia. Esto lo realizaron primero en la ciudad sede del proyecto, Vancouver, y posteriormente en algunas localidades de países como Suecia, Alemania, Italia, Escocia y Francia. Aunado a la grabación en campo, analizaron aquellos aspectos explicativos de esos paisajes sonoros. Para ello consideraron la historia de los lugares, la relevancia cultural de sonidos emblemáticos, cómo ello se relacionaba con los ritmos de la vida social, la acústica de los materiales de calles y edificios y cómo se conformaban lo que llamaron comunidades acústicas, es decir, complejos de sonidos vinculados (World Soundscape Project, 1973; Schafer, 1978). Entre los aportes metodológicos emanados de ello se encuentran las caminatas sonoras 
(World Soundscape Project, 1973). Buena parte de los aportes teóricos derivados de estas investigaciones se encuentran en el Handbook for Acoustic Ecology (Truax, 1978), texto que conjunta las definiciones de los términos usados en sus textos, entre ellas, la de paisaje sonoro, así como la descripción de los métodos que emplearon.

Derivado del interés de Schafer por la problemática de la contaminación acústica, misma que estaba expandiéndose desde las ciudades y zonas industriales a las áreas rurales y naturales, también fue pionero en el ámbito de lo que empezó a llamarse ecología acústica. De acuerdo con Schafer (1977), dicho término refiere al estudio de los efectos de un paisaje sonoro en las formas de vida que lo habitan, generando respuestas físicas y de comportamiento particulares, especialmente cuando esta relación llegaba a un desbalance. Como menciona Barry Truax (2019), en ese entonces se buscó que el paisaje sonoro formara parte de la agenda de los movimientos ambientalistas y, a su vez, que fuera entendido como un sistema complejo de interacciones, más allá de un único fenómeno de lo audible.

Paralelamente, a finales de la década de 1970, en Francia, se formó el Centre de Recherche sur l'Espace Sonore et l'Environnement Urbain (Cresson) en el l'École d'Architecture, en Grenoble. Jean-François Augoyard, uno de los miembros fundadores, conoció la propuesta de paisaje sonoro de Schafer; sin embargo, decidió alejarse de dicho enfoque (Augoyard, citado en Amphoux \& Le Guern, 2017). El grupo académico se enfocó en los ambientes urbanos. Buscaron atender la problemática ambiental del ruido, entendiéndolo desde una postura que no le atribuyó a priori la característica de contaminación; es decir, algunos lugares bulliciosos - como una plaza pública o el mercado - podían tener niveles altos de sonido e interferencias entre ellos; sin embargo, eran aceptados o tolerados culturalmente, en tanto lugares importantes para las dinámicas de ciertas sociedades. A futuro, el Cresson formó escuela, donde se destacan los trabajos de Pascal Amphoux (1993; 2003), quien propuso estudiar el paisaje sonoro a través de las firmas sonoras, las cuales aludían a un sonido o un conjunto de estos que permitían identificar un lugar o momento histórico concreto y, a partir de ello, definir aquellos espacios cuya sonoridad era necesario mantener por su importancia local.

Tras la aparición del World Soundscape Project y el Cresson, el campo de estudio se expandió en términos conceptuales y metodológicos. Las investigaciones de corte ecológico definieron aquellas unidades de análisis para el estudio de la interacción entre organismos y de estos con el ambiente a través del sonido, por ejemplo, nicho acústico y sonotopo. Así mismo, la portabilidad de las herramientas de grabación de audio y con ello la posibilidad de mayores análisis de espectrogramas del sonido permitieron refinar los análisis en la materia. Por otro lado, investigaciones de corte social comenzaron a involucrar a la población residente en las áreas de estudio para comprender la importancia cultural del sonido, como patrimonio y como parte de los aspectos que definen un territorio. Ambas tendencias se desarrollaron de manera independiente y surgieron dos enfoques dentro del campo de estudio del paisaje sonoro: el ecológico y el perceptual-cultural.

\subsection{Expansión del campo de estudio: el enfoque ecológico}

Los aportes del músico y ecólogo estadounidense Bernie L. Krause (1987) fueron claves en el desarrollo del enfoque ecológico. Se enfocó en el análisis de los sonidos producidos por comunidades ecológicas: por ejemplo, las localizadas en el Pico Paradis de la isla de San Martin, en el mar Caribe, donde estudió las vocalizaciones de aves como Chaetura martinica y Zenaida macroura, o en la isla de Borneo en Asia, con las especies Terpsiphone Paradisi, Calorhamphus fuliginosus y Trichastoma bicolor. Su método consistió en el análisis de 
espectrogramas de las grabaciones de audio en estos sitios. Llegó a la conclusión de que las especies habían adaptado sus vocalizaciones a frecuencias acústicas específicas, las cuales no solo respondían a un patrón temporal, sino también a uno espacial, equiparable a lo que se conocía en ecología como nicho.

En este mismo ámbito, en el año 2010, se llevó a cabo un simposio titulado "Soundscape Ecology: The Complexity of Acoustical Patterns in Landscape", en el marco de la reunión anual de la United States Regional Association of the International Association for Landscape Ecology, en la Universidad de Georgia (Truax \& Barret, 2011). Tras dicho encuentro se publicó uno de los artículos más relevantes en la materia, elaborado por Pijanowski y colaboradores (2011). Tomaron como modelo la ecología del paisaje norteamericana (Forman \&
Godron, 1986; Forman, 1995) y propusieron que el paisaje sonoro debía abordarse desde sus procesos y patrones espaciales y temporales y en diferentes escalas. A partir de ello se podían identificar áreas homogéneas dentro de una matriz, así como las interacciones de sus elementos bióticos, abióticos y antrópicos. Propusieron la distinción tipológica de las fuentes de sonido (geofonías, biofonías y antropofonías) (Pijanowski et al., 2011; Truax \& Barret, 2011). Posteriormente, Almo Farina (2014) recopilaría las definiciones respecto a los patrones del sonido según su función y escala (sonotopo, sonotono) (Figura 2). Asimismo, realizaron aportaciones al procedimiento metodológico, tales como las hipótesis de adaptación morfológica, de adaptación acústica, del nicho acústico y del reconocimiento de especies (Pijanowski et al., 2011; Farina, 2014).

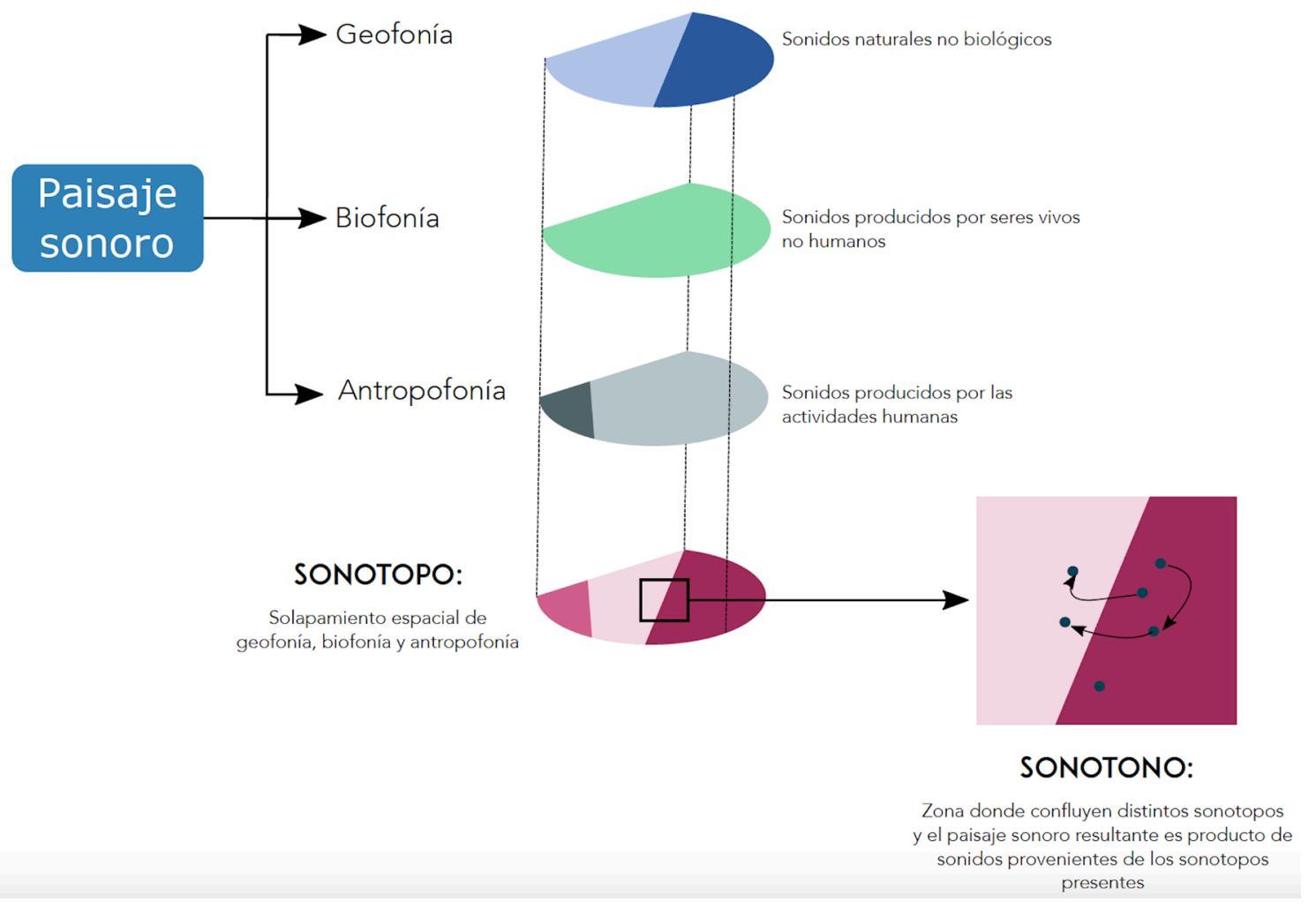

FIGURA 2. Esquema tipológico de las fuentes de sonido de acuerdo con Pijanowski y colaboradores (2011) y patrones espaciales en la ecología del paisaje sonoro de acuerdo con Farina (2014)

FUENTE: elaboración propia. 


\subsection{Expansión del campo de estudio: el enfoque perceptual-cultural}

Durante la década de los 1980 la propuesta del paisaje sonoro se difundió por Asia, Europa y, finalmente, en Latinoamérica. Así, los estudios del paisaje sonoro llegaron a Japón, gracias a Keiko Torigoe. Esta investigadora realizó, a inicios de la década, una tesis de maestría en la Universidad de York, en Toronto, donde detalló la historia del World Soundscape Project y trabajó de cerca con sus miembros (Torigoe, 1982). Por tanto, estaba familiarizada no solo con el concepto de paisaje sonoro, sino también con la metodología de este grupo de trabajo. Torigoe le dio un papel central a la investigación histórica enfocada en el paisaje sonoro. Su método consistió en el análisis de documentos de audio de periodos históricos y entrevistas a las personas de mayor edad en los sitios donde desarrollaba sus investigaciones. Por ejemplo, para la zona de Kanda, en Tokio, determinó que los eventos que implicaron mayores cambios para su paisaje sonoro fueron el terremoto ocurrido en 1923 y los Juegos Olímpicos de Tokio en 1964, donde el segundo evento fue el que configuró su paisaje sonoro actual (Hiramatsu, 1993).

También en Japón surgió como propuesta la soundscapegraphy, término que refiere a una manera de aplicar la etnografía enfocada al sonido y su percepción. En ella se contemplan, como fuentes de información, las observaciones en campo (medición de niveles de sonido y grabación de audio), cuestionarios, entrevistas, revisión documental (novelas, monografias, reportes periodísticos y publicidad) y el análisis de pinturas, fotografías y objetos a través de los cuales se pudieran inferir las sonoridades históricas. Kozo Hiramatsu (2003), autor de la propuesta, agregó que en estudios de esta naturaleza es indispensable la participación de los habitantes del lugar y conocer sus historias de vida; de otra manera, lo registrado no llega a mostrar la complejidad sonora de un lugar.
En España, el músico y ecólogo José Luis Carles formuló una crítica a la modernidad, argumentando que al iniciar la era industrial se presentó un decaimiento en la sensibilidad auditiva, lo cual trajo como consecuencia la pérdida de todo un complejo de señales y marcas acústicas ligado con identidades culturales (Carles, 1992). Posteriormente desarrolló su investigación sobre la importancia del paisaje sonoro como un elemento que determinaba nuestra percepción ambiental (Carles et al., 1999).

En América Latina los trabajos de paisaje sonoro se desarrollaron en la década de los noventa. A ello contribuyó la traducción al español y posteriormente al portugués de obras de Schafer y las visitas que realizó a la región para impartir talleres, como los que se llevaron a cabo entre 1991 y 1992 en las ciudades brasileñas de São Paulo y Londrina. Esto a su vez estimuló la creación del proyecto Paisajes Sonoros y Ecología Acústica de Brasil (El-Haouli, 2008). Por otro lado, en el año de 1999, en Chile, surgió el Programa Paisaje Sonoro a cargo de Luis Barrie, cuya finalidad fue el rescate del patrimonio inmaterial sonoro a través de la indagación en torno a la percepción auditiva de pueblos originarios, específicamente los mapuches y los rapanuis (Barrie, 2008). Otro ejemplo es el proyecto Paisaje Sonoro Uruguay, desarrollado por el Estudio de Música Electroacústica de la Escuela Universitaria de Música, en la Universidad de la República Uruguay, cuyos trabajos iniciaron en el año 2000, cuando documentaron el paisaje sonoro de Montevideo (Maggiolo, 2003), para lo cual retomaron las bases conceptuales y metodológicas del World Soundscape Project.

De manera más reciente, en América Latina, los trabajos de paisaje sonoro han reflexionado en torno a temas como el patrimonio y el territorio. Un ejemplo de ello fue el proyecto Paisajes Sonoros Cafeteros, desarrollado en Colombia durante el año 2013, que, además de la documentación de sonidos como parte del patrimonio inmaterial, buscó generar metodologías de trabajo adecuadas a la realidad regional y 
estimular la conciencia de la riqueza sonora local ante aquellos métodos foráneos generados por los procesos de patrimonialización institucional (Román, 2016). En ese sentido, otras investigaciones enfocadas en las lenguas o géneros musicales tradicionales - considerados elementos que conforman los paisajes sonoros - han documentado la manera en que la preservación, la estimulación y la adaptación de dichas expresiones por distintos grupos culturales les permitió hacer frente a procesos globalizadores y de desterritorialización. Ejemplo de lo anterior es el caso del son "El canario indígena" — pieza musical adaptada del son "El canario", popular en la región Huasteca, ubicada al noreste de México-, con el cual sus autores, al incorporar lirica en lengua náhuatl, buscaron estimular la identidad territorial y abonar a tareas de preservación lingüística (Flores-Martínez \& Figueroa, 2018).

\subsection{Paisaje sonoro y geografía: un acercamiento desde el enfoque cultural}

Las primeras referencias al paisaje sonoro desde el enfoque de la geografía cultural pueden identificarse a finales de la década de los 1980, cuando Douglas Pocock (1989) publicó "Sound and the Geographer" en la revista Geography del Reino Unido, en donde describe las contribuciones que, a su consideración, el estudio del sonido podía aportar a la geografía. Señaló la necesidad de conocer las propiedades del sonido en el espacio, como un elemento que brinda información de los sitios donde se produce. Enfatizó que la experiencia del sonido se determinaba tanto por aspectos personales - las condiciones físicas y de salud de los individuos, así como su historia de vida y sus actividades habituales - como por el contexto en el que el sonido es producido - es decir, su ocurrencia en espacios cerrados o abiertos y los elementos materiales presentes - Además, remitió a las propuestas de Schafer, justificando que eran una buena aproximación a la espacialidad del sonido y una manera adecuada de llegar a caracterizaciones de elementos paisajísticos que de otra forma resultaban dificiles de describir.

Otro trabajo que contribuyó a la discusión desde la geografía cultural fue el de Susan J. Smith (1994), "Soundscape", publicado en la revista Area. En su artículo, Smith formuló una crítica al dominio de lo visual en los estudios de paisaje. Consideró que si el enfoque de la geografía cultural aspiraba a entender el paisaje desde las subjetividades y como un reflejo de la vida social no podía dejar de lado su dimensión audible y las expresiones culturales como la música. Fue enfática en que los trabajos donde se había empleado la etnografía habían dejado en un lugar marginal los sonidos, prácticamente sin documentar — sea de manera escrita o en grabación de audio-, por lo que se había diluido la oportunidad de entender el papel del sonido directamente en su contexto de ocurrencia. Además, consideró a la música como un elemento del paisaje sonoro que podía articular análisis referentes al sentido de comunidad e identidad a escala local, así como referencia de los esfuerzos por el mantenimiento de rasgos locales ante procesos de globalización y mercantilización de prácticas locales.

En el mismo año, Paul Rodaway (1994) publicó el libro Sensous Geography. Body, sense and place, en el cual retomó los planteamientos de Schafer con una mirada crítica, destacando que su propuesta era muy conveniente al momento de realizar descripciones de la ecología acústica; sin embargo, la considera un símil parcial de la noción de paisaje. Por tanto, el planteamiento desde la ecología resultaba limitado por el alto dinamismo de los fenómenos sonoros y sus conectividades energéticas, que dejaban en un segundo plano la experiencia sensorial de las personas en estrecha relación con sus lugares específicos. Propuso entonces el uso del término geografias auditivas, con el cual buscó abordar la manera en que fisiológicamente se percibe el sonido y cómo a partir de ello se desarrollaban capacidades de anticipación, asociación y recuerdos en lugares concretos. 
Otra idea que planteó Rodaway es que las particularidades de las formas en que nos relacionamos con el sonido están mediadas por desarrollos tecnológicos y maneras de interacción con el medio. Por ejemplo, argumentó que en contextos rurales tienen mayor relevancia la tradición oral y la escucha en espacios abiertos, y que esta última permite identificar aspectos ambientales como la localización de fuentes de alimentos o la anticipación de fenómenos climáticos. En contraste, según Rodaway, los elementos visuales tienen mayor importancia en las culturas de contextos mayormente urbanizados e industrializados, donde la amplificación y la mecanización de sonidos homogenizan aquellos elementos que es posible apreciar con el oído (Rodaway, 1994).

Posteriormente, la investigación que realizó David Matless (2005) adquirió notoriedad en los estudios del paisaje sonoro en la geografia cultural. El autor se refirió a su trabajo como una geografía del sonido, lo cual le dio mayor libertad para problematizar los fenómenos sonoros en función de los actores que se desenvuelven en un espacio específico, con la posibilidad de abordar distintas escalas como lo local o regional. En su investigación describió la forma en que históricamente se definió la identidad sonora de la región de Norfolk Broads, Reino Unido, y cómo ello fue un factor que generó disputas por mantener sus características. El autor examinó documentos como estudios acústicos en el sitio, descripciones en guías de viajero, diarios locales, poemas, novelas, canciones, normativas locales respecto del sonido y textos institucionales sobre el área tras ser declarada parque nacional. Con ello logró identificar elementos en común sobre la descripción del paisaje sonoro, que en general exaltan su tranquilidad y la presencia de sonidos naturales. Cuando agentes externos rompían con la permanencia de dichos elementos, por ejemplo, las visitas de turistas, estos eran juzgados y controlados. Su trabajo se consideró innovador, pues mostró la manera en que un paisaje sonoro se asume como elemento de la identidad en escala regional y local, y, a su vez, cómo en esa idea se reproduce y existen esfuerzos para mantenerla desde varios ámbitos: lo ecológico - políticas de conservación-, lo cultural - se promueven elementos específicos como tipos particulares de música- y lo normativo institucional - reglamentos de qué sonidos son aceptados y en qué lugares y momentos-.

\subsection{Panorama actual de los trabajos de paisaje sonoro en geografía cultural}

Los trabajos reseñados en la sección anterior muestran cómo, desde la discusión del paisaje sonoro en la geografía cultural, emanaron propuestas como la geografía auditiva o la geografía del sonido. Por ello, esta sección se refiere a los trabajos que están en la frontera entre esas formas de abordar el sonido dentro de la geografía cultural. Cabe destacar el interés en el tema mostrado por la geografía británica (Figura 3). Una línea de trabajo que se identificó en dicho contexto fueron los estudios con perspectiva histórica. Un aporte interesante de estos trabajos es la manera en que los documentos escritos, ubicados en archivos históricos, han sido retomados como fuente de datos para comprender el papel del sonido en otras épocas.

A partir de las fuentes históricas, Simpson (2017) realizó una descripción del paisaje sonoro de las calles de Londres durante la época victoriana centrándose en la música callejera. Identificó que estas interpretaciones musicales en el espacio público vulneraban otros de índole privada, lo cual desembocó en discusiones que llegaron hasta el Parlamento británico y, consecuentemente, en reglamentaciones al respecto. El autor concluyó que el paisaje sonoro es un medio en el que se conglomeran y expresan una diversidad de contextos de orden social, material y político, los cuales definen la percepción de lo escuchado y las acciones que se desprenden de ello según jerarquías sociales y políticas. 


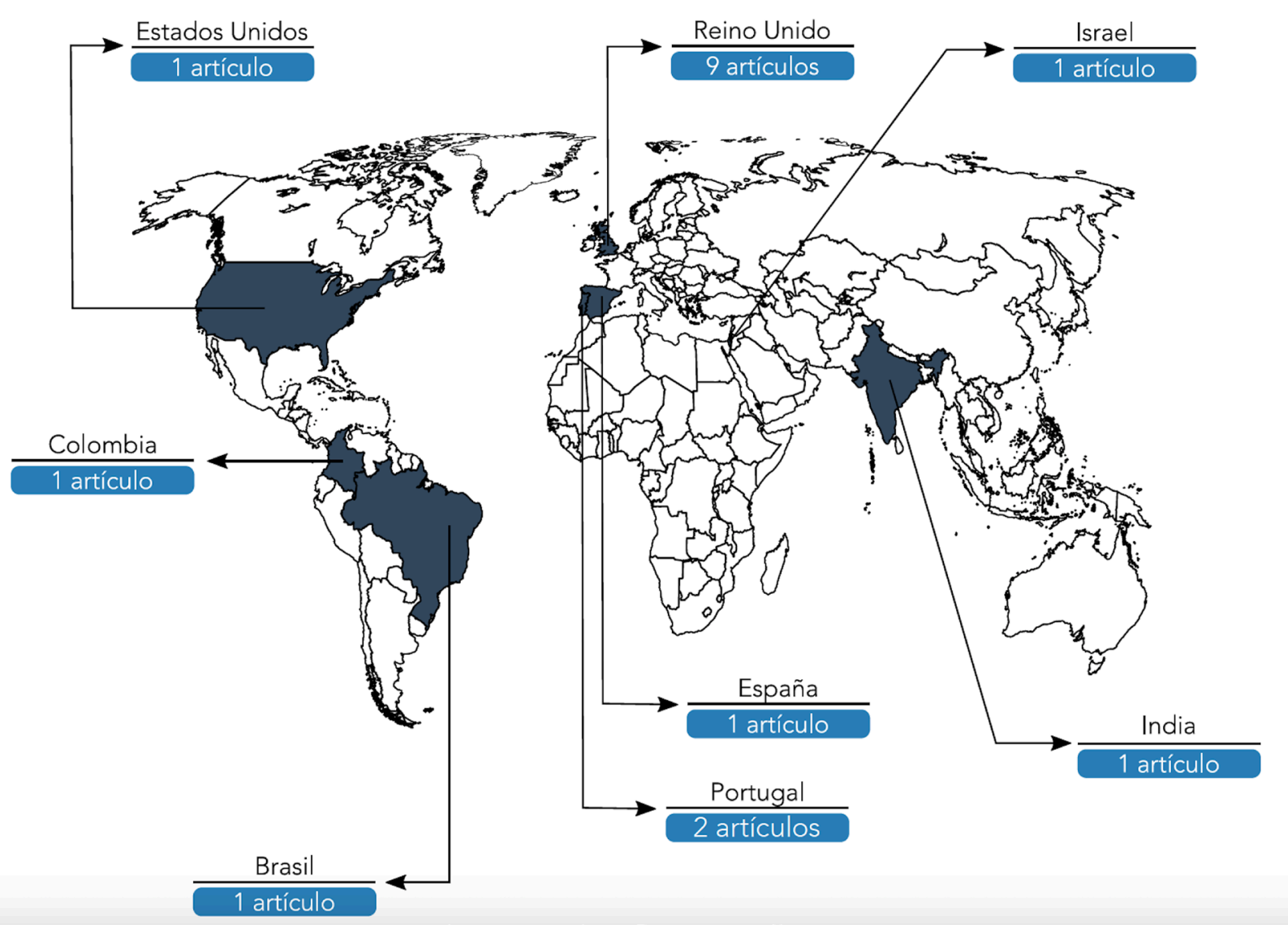

FIGURA 3. Países donde se han realizado investigaciones de paisajes sonoros con enfoque explícito de geografía cultural

FUENTE: elaboración propia.

En un sentido similar, Elin Jones (2020) analizó el papel del sonido en las rebeliones llevadas a cabo por quienes laboraban en las embarcaciones de la Marina Real Británica durante la segunda mitad del siglo XVIII y principios del xIX. Señaló que, en ese contexto, la organización y la comunicación de los marineros mediante sonidos - como gritos, murmullos, silencios y aplausos - fue fundamental para exigir mayores derechos y protestar ante abusos de la tripulación. Aunado a lo anterior, destacó que la geofonía en altamar impedía que los oficiales escucharan claramente lo que ocurría en todas las secciones que conformaban los barcos, lo cual fue un factor a favor de la organización y las rebeliones de los marineros.

Otro estudio de corte histórico fue el que realizó Julian Holloway (2017), en donde aplicó una metodología que denominó "volver a hacer sonar" (resounding). Uno de sus objetivos fue consolidar una estrategia para experimentar nuevamente los sonidos de actividades y sitios importantes de algún lugar para un periodo histórico especifico. En este caso, el trabajo de Holloway buscó recrear el paisaje sonoro del pueblo de Eyam en Derbyshire, Reino Unido, durante el brote de la peste bubónica en el año de 1665. A partir de las fuentes documentales, determinó una serie de sitios experimentales en los que se podrían volver a escuchar sonoridades similares a las del periodo señalado. En palabras del autor, es una manera de hacer audible la historia de un paisaje y con ello explorar vínculos afectivos y memorias a partir de la experiencia directa en los espacios.

Dentro de los trabajos británicos también se identificó una línea interesada en procesos territoriales e identitarios que involucran al paisaje sonoro. Por 
ejemplo, Tariq Jazeel (2005) realizó análisis de los discursos, actividades y espacios vinculados a la música británica con influencias asiáticas e identificó que esto define aquellos espacios que se vuelven parte de la identidad de ciertos grupos. Por su parte, Phillip Boland (2010) trabajó sobre los acentos en la lengua inglesa. Analizó la sonoridad del scouse, dialecto del inglés que identifica a una parte de los habitantes de Liverpool. Concluyó que era un aspecto que llevaba a lo que llamó exclusión sonora, es decir, grupos que pueden ser identificados a partir del sonido - en este caso, su acento - y ser excluidos o excluir ellos mismos.

Siguiendo esta línea de interés, Julia C. Obert (2014) analizó el paisaje sonoro de la ciudad de Belfast, en Irlanda del Norte. La autora mostró cómo se han conformado territorial e históricamente ciertas áreas de la ciudad a través de los sonidos, mismos que a su vez corresponden a elementos de la identidad política y religiosa de los distintos grupos culturales que la habitan. Concretamente, identificó las diferencias entre barrios de irlandeses y protestantes, las cuales se pueden percibir tanto en los sonidos de las actividades que se llevaban a cabo como en la música que se escuchaba en los sitios y las celebraciones públicas que ocurrían. Otro aspecto que estudió fue el simbolismo del sonido en desfiles como el de la Orden de Orange, en el cual los grupos de protestantes reafirmaban su territorialidad no solo con la ruta del contingente, sino también con los sonidos de tambores y flautas.

Entre las discusiones sobre la representación de paisajes sonoros se encuentra el trabajo de George Revill (2014), quien destacó que el sonido en el paisaje es un elemento que transmite información, pero también el medio a través del cual se le da sentido al paisaje. Revill ejemplifica cómo una representación del paisaje en audio cuenta con la capacidad de transmitir sus especificidades históricas, geográficas y culturales. Para ello, analiza un montaje de audio titulado El tren fantasma, realizado por el artista sonoro Chris Watson, el cual se compone de grabaciones tomadas durante un recorrido en tren a través de
México, desde la costa del océano Pacífico hasta el golfo de México. Destaca que la creación de Watson brinda la sensación de movimiento, algo de lo que las representaciones visuales de paisaje carecen.

En discusiones enfocadas a los métodos de estudio del sonido en geografia, Gallagher y Prior (2014) propusieron usar la fonografía como una herramienta de mayor relevancia dentro de la disciplina. Uno de los argumentos centrales que plantearon fue que las grabaciones de audio de los contextos espaciales donde se ha realizado trabajo de campo proporcionaban detalles de actividades cotidianas o lugares relevantes en una localidad que quedaban fuera de los registros textuales y fotográficos; por lo tanto, es necesario implementarla más cotidianamente. Otro elemento que propusieron era la incorporación de la escucha expandida (Gallagher et al. 2017). Dicho término hace referencia a "la variedad de formas en que humanos y más-que-humanos respondemos al sonido" (Gallagher et al., 2017, p. 618).

En España, Benjamin Fraser (2011) reflexionó sobre el papel de la producción musical en contextos urbanos, asumiéndola como contenedora y desencadenante de emociones, así como un factor en el desarrollo de identidad y del sentido de lugar. Usó como objeto de análisis las líricas de canciones de la banda de rock Lisabö, originaria del País Vasco. El autor generó una coyuntura entre el término paisaje sonoro y las propuestas provenientes de otras disciplinas de las ciencias sociales, por ejemplo, el tratamiento de la alienación urbana y el ritmoanálisis de Henri Lefebvre (1992) y los postulados de David Harvey (1996) sobre el lugar. Identificó que en ellas se muestra la identidad político-territorial de sus integrantes y una crítica a la alienación urbana, esta última expresada como un descontento hacia la creciente presencia de sitios que han sido diseñados conforme a patrones que buscan homogenizar espacios y que, en consecuencia, carecen de una carga simbólica y afectiva.

En Portugal, Carlos Fortuna (2009) se propuso analizar cómo la globalización durante el siglo xx ha 
conformado los paisajes sonoros urbanos. Explicó las consecuencias de la llegada del motor de combustión a la cotidianidad urbana, ya que significó el inicio de la invasión de sonidos mecánicos en las ciudades. Con ello inició la globalización sonora, en la cual sonidos singulares a nivel local comenzaron a perder notoriedad o incluso desparecieron ante otros acarreados por avances tecnológicos. En las décadas recientes, describió el autor, este proceso sigue, ahora en la época del beep, los teléfonos celulares y la individualización de la escucha por medio de reproductores de música portátiles. Fortuna pretendió hacer un llamado para reflexionar sobre la importancia del paisaje sonoro como un tema geográfico poco explorado.

En el mismo país, Daniel Paiva (2018) realizó una revisión sistematizada de trabajos de la geografía del sonido en la que incluyó un apartado para el tema de "paisaje, representaciones espaciales e identidad". Un punto que el autor destacó en su revisión sobre ese tema en particular es la falta de trabajos críticos desde la geografía sobre el concepto de paisaje sonoro. Consideró que el trabajo de Rodaway, si bien realizó críticas, adolece de propuestas al respecto y que este es un aspecto importante por desarrollar en investigaciones futuras.

En América Latina, desde la geografía brasileña, Torres y Kozel (2010) analizaron el papel de la música como elemento del paisaje sonoro y como objeto de estudio de la geografía cultural. Plantearon que la identidad de personas y lugares podía analizarse mediante los elementos sonoros del espacio, de los que forman parte el lenguaje, la comunicación y el arte. En este sentido, consideran de importancia los estudios de las composiciones musicales, al ser la expresión audible de los tres elementos mencionados y un claro ejemplo de cómo estos se compaginan. Argumentaron que el enfoque de la geografía cultural permite interpretar la memoria, los símbolos, los valores sociales y las ideas sobre los paisajes que se estudian y representan.

En Colombia, Joaquín Llorca (2017) concretó una definición de territorio sonoro, término con el cual se refirió a un sistema de relaciones delimitado espacialmente que al interior funciona mediante una red de elementos audibles que se expresan con cierta periodicidad, y que en conjunto caracterizan ese espacio. Usó como ejemplo lo que acontece en el barrio de San Nicolas, en Cali. Allí los locales dedicados la industria gráfica y editorial, ubicados en una zona específica de dicha localidad, son los que determinan el paisaje sonoro y sus características, definidas principalmente por el constante sonido de las máquinas que se utilizan en las imprentas. Lo anterior, analizado desde su propia propuesta de territorio sonoro, mostró que en torno a estas labores se configura una red de actividades y sonidos, como el de abrir y cerrar las puertas de los establecimientos, las carretillas con las que se transporta papel y tinta y la música que en esos sitios se suele escuchar.

En Estados Unidos, Brandi T. Summers (2021) analizó el movimiento \#DontMuteDC, surgido en la ciudad de Washington D.C., el cual exigió que se respetaran los espacios en que la música go-go es escuchada e interpretada. La autora explicó que ese género musical es un elemento que ha caracterizado durante más de cuarenta años el paisaje sonoro de la ciudad. Pese a lo anterior, la gentrificación que ha venido ocurriendo ha buscado imponer formas estéticas incluso respecto a los sonidos en espacios públicos, es decir, controlar qué, cómo y cuándo se escucha algo, postura en la que se excluye la música go-go. Esto último fue motivo de protestas para evitar que la tradición del go-go fuera silenciada.

Los trabajos asiáticos también han analizado dinámicas sociales y territoriales a través de una interpretación del paisaje sonoro. Es el caso que presenta Tripta Chandola (2012), quien señaló que existían dinámicas y jerarquizaciones sociales que podían interpretarse a través del paisaje sonoro. Tomó como caso de estudio las dinámicas sociales alrededor de la disponibilidad y el acceso al agua en la localidad de Govindpuri en Delhi, India, y llevó a cabo un trabajo etnográfico, el cual adaptó a cuestionamientos clave como el quiénes 
y cómo están escuchando, así como qué sonidos son aceptados y cuáles ignorados. Chandola concluyó que la escucha estaba determinada por factores sociales, culturales y de género, y, en ese sentido, que el paisaje sonoro también era un reflejo de la articulación y jerarquización interna que existía en las sociedades.

El paisaje sonoro puede ser reflejo y motivo de tensiones existentes entre dos grupos culturales con conflictos históricos y territorios adyacentes. Ejemplo de esto es el caso de estudio que presentó Abigail Wood (2015), donde analiza desde el paisaje sonoro cómo se expresan las identidades religiosas y nacionales en las cercanías del Muro de las Lamentaciones o Muro Occidental en Israel, que se encuentra en la frontera del territorio judío y musulmán en Jerusalén. La autora, a través de un análisis histórico y de trabajo etnográfico, identificó que han existido esfuerzos históricos de regulaciones en torno al sonido generado por estos grupos, lo que ha permitido su coexistencia. Sin embargo, la imposibilidad de limitar espacialmente los sonidos que se producen es una razón para que ocurran actos, en ocasiones involuntarios, que pueden considerarse provocaciones. La autora propuso entender sitios como el Muro de las Lamentaciones como espacios heterofónicos, en los cuales es complicado que no ocurra un solapamiento de los sonidos culturalmente producidos y, por ende, que se generen conflictos.

\section{Conclusiones}

El paisaje sonoro, como campo de estudio, presentó una expansión considerable durante la década de 1980, principalmente en el mundo anglófono e hispanohablante. Desde ese momento es posible identificar dos corrientes principales: la ecológica y la perceptual-cultural. La corriente ecológica se consolidó de manera temprana en la ecología acústica y más recientemente en la ecología del paisaje sonoro. La segunda vertiente, la perceptual-cultural, se vinculó con disciplinas dentro de las ciencias sociales, entre ellas la geografía. En un primer momento, se asumió como una propuesta que enriquecía análisis de fenómenos culturales y sociales (Pocock, 1989; Smith, 1994). Sin embargo, lecturas críticas posteriores optaron por proponer términos con énfasis más claros en cuanto a abordajes geográficos, de donde emanaron las geografias auditivas (Rodaway, 1994) y las geografías del sonido (Matless, 2005).

La revisión bibliográfica específica para la geografía cultural mostró que se ha mantenido latente un interés por incorporar el sonido como objeto de estudio a través del paisaje sonoro, las geografias auditivas y las geografias del sonido. En este punto es necesario señalar que la forma en que se desarrolló el procedimiento metodológico, enfocado en dos índices y dos buscadores de literatura académica, resulta limitado. La razón es que no se ven representadas las investigaciones desarrolladas fuera del mundo anglófono y de Europa occidental. Complementar este ejercicio de revisión desde otros motores de búsqueda y en otras locaciones podría dar cuenta de posturas innovadoras, grupos de investigación que han enriquecido el campo de estudio del paisaje sonoro o que analizan la forma en que es valorado y significado en culturas distintas a la occidental, por ejemplo, lo desarrollado en Latinoamérica en cuestiones de género (Gutiérrez-Rojas, 2015), de reinterpretación del paisaje sonoro como obra musical (Aguilar-López, 2020), las diversas experiencias de mapeo sonoro por toda la región (Archivo Usted no está aquí, 2021) o su articulación con la antropología a través de la acustemología (Galván-Cázarez \& Rivera-González, 2021).

Finalmente, la revisión presentada es apenas un esbozo de los trabajos que desde la geografía cultural contemplan el sonido como objeto de estudio. Sin embargo, es posible afirmar que el paisaje sonoro se ha convertido en una noción que empuja la reflexión teórica y metodológica de los estudios culturalistas en geografía y que hoy en día se ha vuelto un medio para explorar la historia, las identidades, las percepciones, las disputas e incluso nuevas formas para representar el espacio más allá del texto y el mapa convencional. 


\section{Referencias}

Aguilar-López, E. E. (2020). MusiCultura Huasteca. Trabajos de creación artística multidisciplinar en torno a la Huasteca. En P. Ayala-García (coord.), Sincretismo artístico. Las Artes, sus discrepancias, concordancias y una docencia conciliadora (pp. 19-25). Universidad de Colima.

Amphoux, P. (1993). Sound signatures, configurations and effects. Architecture \& Comportement / Architecture \& Behaviour, 9(3), 387-395.

Amphoux, P. (2003). L'Identite sonore urbaine. Une approche méthodologique croisée. En G. Moser \& K. Weiss (Eds.), Espacies de vie: aspects de la relation homme-environnement (pp. 85-112). Armand Colin.

Amphoux, P. \& Le Guern, P. (2017). Le temps des pionniers: Jean-Fraçois Augoyard et le Cresson. Politiques de communication, 1(HS), 31-44. https://doi.org/10.3917/ pdc.hs01.0031

Antrop, M. \& Eetvelde, V. V. (2019). Territory and/or scenary: concepts and prospects of western landscape research. In L. Mueller \& F. Eulenstein (eds.), Current Trends in Landscape Research (pp. 3-39). Springer. https://doi.org/10.1007/978-3-030-30069-2

Archivo Usted no está aquí (2021). Mapas sonoros de Latinoamérica. https://archivoustednoestaaqui.bandcamp. com/album/mapas-sonoros-de-latinoam-rica-i-transformaci-n-sonora-en-confinamiento-compilado-1

Barnett, C. (1998). Cultural twists and turns. Environment and Planning: Society and Space, 16(6), 631-634.

Barrie, L. (2008). Patrimonio, oralidad y paisaje sonoro. En J. L. Carles (coord.), I Encuentro Iberoamericano de Paisajes Sonoros. Instituto Cervantes, Orquesta y Coro Nacionales de España. https://cvc.cervantes.es/ artes/paisajes_sonoros/p_sonoros01/barrie/barrie_01.htm

Boland, P. (2010). Sonic geography, place and race in the formation of local identity: Liverpool and Scousers. Geografiska Annaler. Series B, Human Geography, 92(1),1-22. https://doi.org/10.1111/j.14680467.2010.00330.x

Carles, J. L. (1992). Nuestra memoria sonora: importancia de los archivos sonoros. Historia y Fuente Oral, 7, 189-191.

Carles, J. L., López-Barrio, I., \& Lucio, J. V. (1999). Sound influence on landscape values. Landscape and Urban Planning, 43(4), 191-200. https://doi.org/10.1016/ S0169-2046(98)00112-1
Chandola, T. (2012). Listening in to water routes: Soundscapes as cultural systems. International Journal of Cultural Studies, 16(1), 55-69. https://doi. org/10.1177/1367877912441436

Claval, P. (1999). Los fundamentos actuales de la geografía cultural. Documents d'Anàlisi Geogràfica, 34, 25-40.

Cosgrove, D. (2002). Observando la naturaleza: el paisaje y el sentido europeo de la vista. Boletín de la Asociación de Geógrafos Españoles, 34, 63-89.

Eden, S. (2004). Greenpeace. New Political Economy, 9(4), 595610.https://doi.org/10.1080/1356346042000311191

El-Haouli,J. (2008). Paisajes sonoros de Brasil: experiencias interdisciplinarias. En J. L. Carles (Coord.), I Encuentro Iberoamericano de Paisajes Sonoros. Instituto Cervantes, Orquesta y Coro Nacionales de España. https://cvc. cervantes.es/artes/paisajes_sonoros/p_sonoros01/ haouli/haouli_01.htm

Farina, A. (2014). Soundscape ecology. Principles, patterns, methods and applications. Springer. https://doi.org/10.1007/97894-007-7374-5

Ferreti, F. (2019). Rediscovering other geographical traditions. Geography Compass, 13(3), e12421. http://doi. org/10.1111/gec3.12421

Flores-Martínez, J. A. \& Figueroa, M. (2018). Procesos de resistencia cultural y mantenimiento del paisaje sonoro: el caso del son El canario indigena. Trans. Revista Transcultural de Música, 21-22. https://www.sibetrans. com/trans/public/docs/2-trans-2018.pdf

Forman, R. T. T. (1995). Land mosaics. The ecology of landscape and regions. Cambridge University Press.

Forman, R. T. T. \& M. Godron (1986). Landscape Ecology. John Wiley \& Sons.

Fortuna, G. (2009). La ciudad de los sonidos: una heurística de la sensibilidad en los paisajes urbanos contemporáneos. Cuadernos de Antropología Social, 30, 39-58. https:// www.redalyc.org/pdf/1809/180913916003.pdf

Fraser, B. (2011). Re-scaling emotional approaches to music: Basque band Lisabö and the soundscapes of urban alienation. Emotion, Space and Society, 4(1), 8-16. https://doi.org/10.1016/j.emospa.2010.03.002

Gallagher, M. \& Prior, J. (2014). Sonic geographies: exploring phonographic methods. Progress in Human Geography, 38(2), 267-284. https://doi. org/10.1177/0309132513481014

Gallagher, M., Kanngieser, A., \& Prior, J. (2017). Listening geographies: landscape, affect and geotechnologies. Progress in Human Geography, 41(5), 618-637. https:// doi.org/10.1177/0309132516652952 
Galván-Cázarez, G. A. \& Rivera-González, J. G. (2021). La escucha en la ciudad contemporanea. Aproximaciones teóricas. Decumanus Revista Interdisciplinaria sobre Estudios Urbanos, 7(7), 48-62. https://doi.org/10.20983/ decumanus.2021.2.3

Gutiérrez-Rojas, M. A. (2015). Música, memoria y resistencia de la mujeres en Bogotá. [Tesis de grado]. Pontificia Universidad Javeriana. https://repository.javeriana.edu.co/ handle/10554/20568

Harvey, D. (1996). Justice, Nature and the Geography of Difference. Blackwell.

Hiernaux, D. (2010). La geografia hoy: giros, fragmentos y nueva unidad. En A. Lindón \& D. Hiernaux (eds.), Los giros de la geografia humana. Desafios y horizontes (pp. 43-61). Anthropos/uam-I.

Hiramatsu, K. (1993). Some aspects of soundscape studies in Japan. Fournal of the Acoustic Society of Japan, 14(3), 133-138.

Hiramatsu, K. (2003). The idea of soundscapegraphy and its description of old Town of Kyoto with Gion Festival. Conference proceedings from the Australian Forum for Acoustic Ecology/World Forum for Acoustic Ecology, 19-23 de marzo, Australia.

Hobsbawn, E. (1995). Historia del siglo xx, 1914-1991. Crítica.

Holloway, J. (2017). Resounding the landscape: the sonic impress of and the story of Eyam, plague village. Landscape Research, 42(6), 601-615. https://doi.org/1 0.1080/01426397.2017.1315387

Jazeel, T. (2005). The world is sound? Geography, musicology and British-Asian soundscapes. Area, 37(3), 233-241. https://doi.org/10.1111/j.14754762.2005.00626.x

Jones, E. (2020). Space, sound and sedition on the Royal Naval ship, 1756-1815. Fournal of Historical Geography, 70, 65-73. http://doi.org/10.1016/j.jhg.2020.07.004

Kramsch, O. (1999). El horizonte de la nueva geografía cultural. Documents d'Anàlisi Geogràfica, 34, 53-68.

Krause, B. L. (1987) The Niche Hypothesis: How Animals Taught Us to Dance and Sing. Whole Earth Review, 57, $1-6$.

Lefebvre, H. (2004). Rhythmanalysis: space, time and everyday life. Continuum.

Llorca, J. (2017). Paisaje sonoro y territorio. El caso del barrio San Nicolás en Cali, Colombia. Revista INVI, 32(89), 9-59. http://dx.doi.org/10.4067/S07 1883582017000100009
Maggiolo, D. (2003). Soundscape research in Uruguay. Soundscape, 4(1), 51-52.

Marontate, J., Robertson, M., \& Clarkson, N. (2016). Soundscape as commemoration and imagination of the acoustic past. In A. L. Tota \& T. Hagen (eds.), Routledge International Handbook of Memory Studies (pp. 519-532). Routledge.

Matless, D. (2005). Sonic geography in a nature region. Social \& Cultural Geography, 6(5), 745-766. https://doi. org/10.1080/14649360500258245

Mitchell, D. (1995). Thre's no such things as culture: towards a reconceptualization of the idea of culture in geography. Transactions of the Institute of British Geographers, 20(1), 102-116. https://doi.org/10.2307/622727

Obert, J. C. (2014). "Shared space": A Belfast soundscape study. New Hibernia Review/Irish Éreannach Nua, 18(1), 13-29.

Olwig, K. R. (2019). The meanings of landscape. Essays on place, space, environment and justice. Routledge.

Paiva, D. (2018). Sonic geographies: Themes, concepts, and deaf spots. Geography Compass, 12(7), e12375. https://doi.org/10.1111/gec3.12375

Pijanowski, B. C., Villanueva-Rivera, L. J., Dumyahn, S. L., Farina, A., Krause, B. L., Napoletano, B. M., Gage, S. H., \& Pieretti, N. (2011). Soundscape ecology: The science of sound in the landscape. BioScience, 61(3), 203-216. https://doi.org/10.1525/bio.2011.61.3.6

Pocock, D. (1989). Sound and the geographer. Geography, 74(3), 193-200.

Revill, G. (2014). El tren fantasma: Arc of sound and the acoustics space of landscape. Transactions of the Institute of British Geographers, 39(3), 333-334. https://doi. org/10.1111/tran. 12034

Rodaway, P. (1994). Sensous Geographies. Body, sense and place. Routledge.

Román, G. G. (2016). Paisajes sonoros cafeteros: ecología, patrimonio y territorio. En C. I. Mora-Forero (ed.), Hablemos de diseño industrial (pp. 163-165). Universidad Jorge Tadeo Lozano.

Rose, G. (2003). On the need to ask how, exactly, is geography "visual"? Antipode, 35(2), 212-221. https://doi. org/10.1111/1467-8330.00317

Schafer, R. M. (1967). Ear cleaning. Notes for an experimental music course. Clark \& Cruicksank.

Schafer, R. M. (1977). The soundscape. Our sonic environment and the tuning of the world. Destiny Books. 
Schafer, R. M. (1978). Five Village Soundscape. A. R. C. Publications, World Soundscape Project.

Schafer, R. M. (2006). Hacia una educación sonora. Consejo Nacional para la Cultura y las Artes, Dirección General de Publicaciones Radio Educación.

Simpson, P. (2017). Sonic affects and the production of space: 'Music by handle' and the politics of street music in Victorian London. Cultural Geographies, 24(1), 89109. https://doi.org/10.1177/1474474016649400

Smith, S. J. (1994). Soundscape. Area, 26(3), 232-240.

Summers, B. T.(2021). Reclaiming the chocolate city: Soundscapes of gentrification and resistence in Washington, DC. Environment and Planning D: Society and Space, 39(1), 30-46. https://doi.org/10.1177/0263775820978242

Taylor, K. (2008). Landscape and Memory. Unesco (ed.), Proceedings of the $3^{\text {rd }}$ International Memory of the World Conference, 19-22 de febrero, Australia.

Thompson, E. A. (2002). The soundscape of modernity. Architectural acoustics and the culture of listening in America, 19001933. мIT Press.

Torigoe, K. (1982). A study of the World Soundscape Project [Tesis de maestría, York University]. The Sonic Research Studio World Soundscape Project Database. http:// www.sfu.ca/sonic-studio-webdav/index2.html

Torres, M. A. \& Kozel, S. (2010). Paisagens sonoras: possíveis caminhos aos estudos culturais em geografia. RA'E GA - O Espaco Geografico em Analise, 20, 123-132. http://dx.doi.org/10.5380/raega.v20i0.20616
Truax, B. (1978). Handbook for acoustic ecology. A. R. C. Publications, World Soundscape Project.

Truax, B. (2019). Acoustic ecology and the World Soundscape Project. In M. Droumeva \& R. Jordan (eds.), Sound, Media, Ecology (pp. 21-44). Palgrave Macmillan. https://doi.org/10.1007/978-3-030-16569-7

Truax, B. \& Barret, G. W. (2011). Soundscape in a context of acoustic ecology and landscape ecology. Landscape Ecology, 26, 1201-1207. https://doi.org/10.1007/ s10980-011-9644-9

Urquijo, P. S. (2021). Geografía cultural en los estudios de paisaje en México. En F. Fernández-Christlieb (ed.), El petate y la jicara. Los estudios de paisaje y geografia cultural en México (pp. 105-133). Editions Hispaniques Institut d'Etudes Ibériques et Latino-Américaines.

Urquijo, P. S. \& Barrera, N. (2009). Historia y paisaje. Explorando un concepto geográfico monista. Andamios, Revista de Investigación Social, 5(10), 227-252. http:// dx.doi.org/10.29092/uacm.v5i10.175

Wood, A. (2015). The cantor and the muezzin's duet: Contested soundscapes at Jerusalem's Western Wall. Contemporary Jewry, 35(1), 55-72. https://www.researchgate.net/publication/276899258_The_Cantor_and_The_Muezzin's_Duet_Contested_Soundscapes_At_Jerusalem's_Western_Wall

World Soundscape Project. (1973). The Vancouver Soundscape. Simon Fraser University. 